\title{
A New Strategy for Elimination of Human Paplloma Virus-related Disease after Human Papillomavirus Vaccines Introduction
}

\author{
Yoo-Jin Park, Tae-Hee Kim ${ }^{1}$, Hae-Hyeog Lee ${ }^{1}$ Jun-Mo Kim ${ }^{2}$ \\ Department of Interdisciplinary Programme in Biomedical Science, Soonchunhyang University Graduate School, Asan, ${ }^{\text {Department of }}$ \\ Obstetrics and Gynecology, ${ }^{2}$ Department of Urology, Soonchunhyang University Buchoen Hospital, Soonchunhyang University College of \\ Medicine, Bucheon, Korea
}

Human papillomavirus (HPV) infections are the most common sexually transmitted diseases in male and female. The most common HPV types include HPV 6/11/16/18/31/33/45/52/58. Bivalent (bHPV), quadrivalent (qHPV), and nonavalent (nHPV) vaccines, have been developed worldwide to protect against HPV infections. This paper describes the worldwide trends in HPV infections after HPV vaccination and shows the progressive changes in national HPV vaccination programs toward gender-neutral vaccination (GNV). This review article is based on original and review articles on the HPV-associated disease burden and nationwide vaccination trends. In Korea, epidemiology studies have shown a decline in the incidence of cervical cancer from $16.3 \%$ (in 1999) to $9.1 \%$ (in 2015). Interestingly, based on the 2019 annual report from Korean Center for Disease Control, the incidence of anogenital warts increased in both sexes during 2014-2018 and was 2.6 times higher in male than female in 2018. These results indicate the good efficacy of HPV vaccinations against both diseases in female. Other studies suggest that GNV would almost eliminate the risks of HPV-associated diseases in most countries, compared to female-only vaccination. HPV vaccine coverage remains lower in male in Korea despite the vaccine efficacy in both sexes and the worldwide GNV trend. Scaling up HPV vaccination in both sexes should be encouraged. Furthermore, removing the HPV-attributable burden through nHPV vaccination could be considered, showing that HPV 31, 33, 45, 52, 58, which are not covered by bHPV and qHPV, are as prevalent as HPV 6, 11, 16, 18.

Keywords: Papillomavirus infections; Condylomata acuminata; Papillomavirus vaccines; Sexually transmitted disease, viral

Copyright $\subset$ 2020, Korean Association of Urogenital Tract Infection and Inflammation. All rights reserved. (c) (8) This is an open access article distributed under the terms of the Creative Commons Attribution (c) (1) \&) Non-Commercial License (http://creativecommons.org/licenses/by-nc/4.0) which permits unrestricted non-commercial use, distribution, and reproduction in any medium, provided the original work is properly cited.
Received: 2 December, 2020

Accepted: 17 December, 2020

Correspondence to: Jun-Mo Kim

(iD) https://orcid.org/0000-0003-4978-1867

Department of Urology, Soonchunhyang University Bucheon Hospital, 170 Jomaru-ro, Wonmi-gu, Bucheon 14584, Korea

Tel: +82-32-621-5464, Fax: +82-32-621-5016

E-mail: urojun@schmc.ac.kr

\section{INTRODUCTION}

Human papillomavirus (HPV) infections are the most common sexually transmitted infections in female and male. HPV infections are typically transient without symptoms and are cleared within 1-2 years [1]. On the other hand, persistence can result in anogenital warts (AGW) and premalignant or cancerous lesions in the anogenital and oropharyngeal regions [2,3]. In female, cervical cancer is the most common manifestation, where $100 \%$ of cases are caused by HPV infections. In male, cancers of the penis, anus, oral cavity, and pharynx are associated with HPV. 
Table 1. Currently available HPV vaccinations in Korea

\begin{tabular}{llll}
\hline Characteristic & \multicolumn{1}{c}{ Bivalent (2vHPV) } & Quadrivalent (4vHPV) & Nanovalent (9vHPV) \\
\hline Brand name & Cervarix & Gardasil & Gardasil 9 \\
Manufacturer & GlaxoSmithKline & Merck and Co, Inc. & Merck and Co, Inc. \\
$\begin{array}{l}\text { Virus-like particles } \\
\text { Injection interval }\end{array}$ & 16,18 & $6,11,16,18$ & $6,11,16,18,31,33,45,52,58$ \\
Age of $9-13$ & & 0,6 month & \\
Age $>14$ & & $0,2,6$ month & \\
Age of $9-14$ & 0,6 month & & 0,6 month \\
Age $>15$ & $0,1,6$ month & 0.5 ml & 0,6 month \\
Volume per dose & 0.5 ml & Intramuscular & 0.5 ml \\
Administration & Intramuscular & Intramuscular \\
\hline
\end{tabular}

HPV: human papillomavirus.

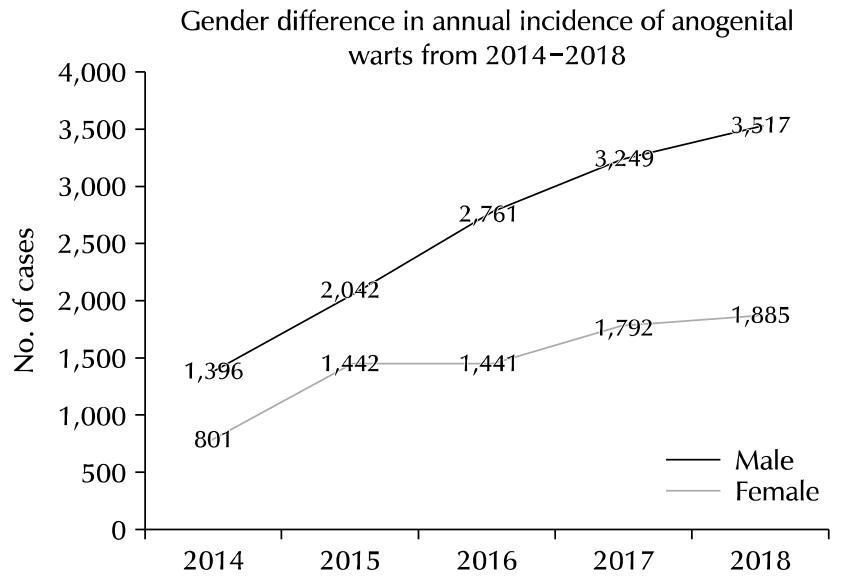

Fig. 1. Tendency of gender difference in anogenital warts prevalence in Korea, 2014.2018 (adapted from 2019 annual report from the Korean Center for Disease Control: Trend of occurrence of Korean domestic sexually transmitted disease declaration) [9].

Three prophylactic HPV vaccines have been licensed worldwide to protect against HPV infections and associated lesions in the cervix, vulva-vagina, and anus: bivalent (bHPV), quadrivalent (qHPV), and nonavalent (nHPV), which target HPV 16/18, HPV 6/11/16/18, and HPV 6/11/16/18/31/33/ 45/52/58, respectively (Table 1). Most national HPV vaccination programs worldwide have been targeting females, with some countries (e.g., United States, Australia, Germany, and United Kingdom) performing gender-neutral vaccination (GNV) [4-6]. Recent studies, however, have suggested that HPV vaccination in both sexes is expected to result in even greater reductions in HPV-associated disease burdens. GNV is expected to result in the near-elimination of AGW in both sexes in Australia, with reductions of more than $90 \%$ in both sexes by 2030 [7]. Eighty percent GNV coverage could eliminate the qHPV types [6].

Although the disparity between the sexes is narrowing worldwide, HPV vaccination in Korea tends to target females. In Korea, reductions in the prevalence of AGW in female have been reported since 2012, which were attributed to the introduction of HPV vaccination [8]. Furthermore, the Korean Center for Disease Control 2019 annual report suggested that the incidence of AGW increased 2.3-3.3 times in both sexes during 2014-2018, and 2.6 times higher in male than female in 2018 (Fig. 1) [9]. Similarly, the nationwide incidence of cervical cancer in Korea has decreased from $16.3 \%$ in 1999 to $9.1 \%$ in 2015 [10]. Nevertheless, new genotypes of HPV other than qHPV types are considered important (e.g., HPV 52, 58) based on epidemiological data, and AGW has been increasing in male in Korea [8]. This review describes the trends in HPV-associated diseases and cancers worldwide and in Korea after introducing HPV vaccination. In addition, the worldwide progressive changes in national programs toward GNV and the necessity of GNV for HPV elimination are described.

\section{MAIN BODY}

\section{Literature Search Strategy and Selection Criteria}

A literature search of electronic databases was conducted using the following keywords: "Human Papillomavirus Infections", and "Papillomavirus Vaccines". These were combined with the following search terms: "Genital Warts", "Cervical Cancer”, "Penile Cancer”, "Oropharyngeal Cancer”, and "Viral Sexually Transmitted Disease" for HPV infections; and "Gardasil" and "Cervarix" for HPV vaccinations.

Original and review articles written in English or Korean were included. Abstract-only studies, case reports, letters to the editors, commentaries, laboratory science studies, and any other non-relevant studies were excluded. The abstracts 
of all identified studies were reviewed to exclude irrelevant articles. Full-text reviews were performed to determine if the inclusion criteria had been satisfied. The reference lists of relevant articles from the first literature search were also reviewed for additional articles.

\section{The Trend of HPV Infections in Korea 1) Prevalence of HPV infections in Korea}

More than 210 HPV genotypes have been officially recognized, some of which are carcinogenic. The most well-known carcinogenic HPV types are 16 and 18. The overall prevalence of HPV infections in Korea ranges from $16.7 \%$ to $40.7 \%$, and HPV 16 is the most common carcinogen [11]. In female worldwide, the most commonly reported HPV type is HPV 16, and the next most common types vary according to the region: HPV 52/58/18 in Asia and HPV $53 / 52 / 18$ in North America [12]. The majority of HPV-associated cancers in male are attributable to HPV 16 $[13,14]$. On the other hand, the prevalence of HPV in Korea has changed.

Nah [11] reported that among the carcinogen types in 18,815 Korean female, the most commonly detected HPV types were $52(3.2 \%), 58(2.7 \%), 16(2.0 \%), 56(1.9 \%)$, and 51 (1.8\%). Lee et al. [15] reported the HPV type distribution among 60,775 female aged $18-79$ years. Among $87.7 \%$ of HPV single-type infections and $12.3 \%$ of multiple infections, the four most common high-risk HPV genotypes were HPV 16 (26.0\%), HPV 52 (25.5\%), HPV 58 (12.3\%), and HPV 18 (7.1\%). According to a study on the HPV-type distribution in five Asian countries (South Korea, Malaysia, Vietnam, Singapore, and the Philippines) [16], the most common types among invasive cervical cancer were HPV 16 (36.8-61.3\%), 18 (12.9-35.4\%), 52 (5.4-10.3\%), and 45 (1.5-17.2\%). In cases of cervical intraepithelial neoplasia $2 / 3$ /adenocarcinoma in situ, HPV 16 (29.7-46.6\%) was the most prevalent, followed by HPV 52 (17.0-66.7\%) and HPV 58 (8.6-16.0\%). The authors reported that HPV 53 (9.69\%), followed by HPV 58 (7.90\%) and $52(7.81 \%)$, are common HPV types, and reported that in Korea, HPV 52 and 58 were prevalent HPV types, as well as HPV 16. Park et al. [17] reported that the most common high-risk HPV types included HPV 16 (6.8\%), HPV 33 (4.5\%), HPV 18 (2.3\%), and HPV 68 (2.3\%) among 150 male patients with AGW. According to a study on the prevalence of HPV in female $(\mathrm{N}=672)$ and male $(\mathrm{N}=381)$ university students in Korea [18], the most common types in female students were
HPV 51, followed by HPV 53 and 56, HPV 16 and 52, and HPV 18. This pattern was similar in male students except for no HPV 56 infections. These HPV types are associated with most HPV-associated diseases.

\section{2) Trend in HPV-associated diseases}

(1) HPV associated diseases in male: HPV infections have various genitourinary manifestations ranging from AGW to penile and anal carcinoma in male [19]. The overall prevalence of AGW in Korea has increased steadily from 51.1 (in 2007) to 98.0 (in 2015) per 100,000, with an annual percent change (APC) of $+8.3 \%$. The prevalence in males has increased steadily over time with an APC of $+11.6 \%$ ( $p$ $<0.001$ ) [8]. Although neonatal uncircumcision is the most significant risk factor for penile cancer, HPV infections are also believed to be associated with penile cancers [20]. In a recent epidemiological study, an increased risk of penile cancer was associated with a history of Bowen's disease (relative risk [RR] 9.4), which is generally considered to be caused by high-risk HPV infections and AGW (RR 5.9). The risk of penile cancer in uncircumcised male (RR 3.2) or circumcised after the neonatal period (RR 3.0) has decreased [21].

The most prevalent HPV infection sites in male are reportedly the penis and scrotum $[22,23]$. Previous studies have reported that the prevalence of HPV infections on the external genitalia ranges from $28-45 \%$ in males aged $17-70$ years [24-26]. According to a recent report, the prevalence of HPV in the external genitalia among 1,160 healthy male aged 18-70 years in Brazil, Mexico, and the United States was $65.2 \%$ in the penile glans, coronal sulcus, penile shaft, and scrotum [27]. Rubin et al. [20] evaluated the prevalence of HPV subtypes in penile intraepithelial neoplasia (PIN), which is a dysplastic premalignant lesion; $90 \%$ of PIN lesions were HPV-positive, with HPV 16 being the most common type (40.7\%). A systematic review of 31 studies, including 1,466 penile carcinomas, revealed a worldwide HPV prevalence of $46.9 \%$, with a relative contribution of HPV 16 (60.23\%), HPV 18 (13.35\%), and HPV 6/11 (8.13\%) [28].

These results suggest a close association between HPV infections and the development of AGW and penile carcinoma. Considering the increasing epidemiological burden of HPV infections in male, HPV is also an important issue for male's health in Korea.

(2) HPV-associated diseases in female: In female, cervical 
HPV infections the most common sexually transmitted infections. Cervical cancer is ranked second in terms of incidence (after breast cancer) and third for mortality (after breast and lung cancers) among female worldwide [29]. Cervical cancer is the seventh most common cancer and the third leading cause of cancer-related death among Korean female [30]. Cervical cancer is the third most common cancer site in female aged 15-34 after thyroid and breast cancer. On the other hand, the nationwide incidence of cervical cancer in Korea has decreased continuously from $16.3 \%$ in 1999 to $9.1 \%$ in 2015, with an estimated APC of $-3.7 \%(\mathrm{p}<0.05)[10]$. Despite the decreasing incidence of cervical cancer, its risks remain high in Korea. The HPV is closely associated with the transformation of cervical epithelial cells, leading to precancerous lesions or cancer [31]. Nah et al. [11] reported that HPV 16, 52, 58, 33, and 31 were prevalent in high-grade squamous intraepithelial lesions (HSIL) in cervical specimens among Korean female. A meta-analysis also found that HPV 58, 18, 52, and 33 were prevalent in HSIL in Korean female [32]. These HPV types can cause AGW or precancerous lesions (HSIL), which may become cancerous. Thus, these HPV types are of concern. For these reasons, many countries have implemented large-scale vaccination projects that have been effective and lifesaving against cervical cancer.

\section{HPV Vaccination}

\section{1) Trend of gender-neutral HPV vaccination globally}

More than 65 countries have introduced HPV vaccination programs. Most of these programs targeted females. In contrast, some countries vaccinate both sexes, including the United States, Australia, Austria, and Switzerland [6]. From 2011 to 2016, the HPV vaccine coverage rates of routine vaccination at age 11-12 years has increased from $37.7 \%$ to $45.7 \%$ in females and from $7.8 \%$ to $27.4 \%$ in males in the United States [33]. The National Cancer Institute (NCI)-designated cancer centers promote practices to eliminate HPV-associated cancers (e.g., cervical, oropharyngeal, anal, and penile cancers) via GNV and cancer screening. They stated that more than $80 \%$ of males and females aged $13-15$ years should be vaccinated by 2020 as part of the nation's Healthy People 2020 goals [34].

The introduction of GNV will decrease the risks of HPV-associated diseases significantly compared to female-only vaccination in most countries. A meta-analysis
[6] predicted that if both sexes are vaccinated, $80 \%$ coverage would be sufficient to eliminate the most common four types of HPV in most models. Eighty percent HPV vaccine coverage in males and females in combination with maintaining long-term vaccine efficacy could eliminate HPV 6, 11, 16, and 18 , with a median relative reduction in HPV prevalence of 1.00 after 70 years in 10 high-income regions, including Australia, Canada, the United Kingdom, and the United States.

Korostil et al. [7] also indicated that GNV would lead to the near-elimination of AGW in both sexes in Australia, with relative reductions of more than 90\% in both sexes by 2030 . These reductions would be less than $80 \%$ and $65 \%$ in females and males, respectively, without male vaccination.

\section{2) HPV vaccination in Korea compared to the global trend}

In Korea, bHPV 16/18 and qHPV 6/11/16/18 vaccines have been available since 2008 and 2007, respectively. Although the nHPV vaccine is available in Korea, it has not been included in the national immunization program (NIP) until now. These vaccines have decreased the prevalence of HPV-associated diseases, such as AGW and cervical cancer. Two-dose HPV vaccines to girls aged 13 or 14 years have been included in the NIP from 2016. On the other hand, the rate of HPV vaccination among males is lower than females in Korea despite the narrowed sex disparity in HPV vaccinations worldwide.

Recently, a nHPV was licensed and launched worldwide, including Korea. In Korea, the nHPV was licensed in January 2016 and was available in July 2016. The nHPV is recommended in America and Europe for the prevention of AGW, precancerous lesions, and cancers of the cervix/vulvo-vagina/anus. In addition to HPV 6, 11, 16, and 18, it covers the high-risk HPV types of HPV 31, 33, 45, 52, and 58, which are not included in bHPV and qHPV vaccines. Although HPV 52 and 58 are prevalent HPV types (in addition to HPV 16) in Korea, they are not covered by the bHPV and qHPV vaccine [35].

Most countries currently only implement vaccination in females. On the other hand, an awareness of the burden of HPV attributable diseases in male has increased. Recent data suggest that it is possible to prevent HPV-associated diseases when implementing GNV. Vaccination in males provides a herd effect in females by preventing HPV spread, as well as personal benefit. These benefits are expected to 
be enhanced using nHPV [36].

\section{3) Effectiveness of HPV vaccination}

(1) Decrease in HPV prevalence and disease after HPV

vaccination in females: Previous studies have suggested that HPV vaccination programs decrease HPV infections in both sexes worldwide. Machalek et al. [37] reported a 92\% decrease in the prevalence of qHPV types in young female aged 18-24 in Australia, from $22.7 \%$ (pre-NIP era) to $1.5 \%$ (8-9 years post-NIP era). This result is consistent with the findings by Garland et al. [38], who evaluated the prevalence of qHPV vaccine-targeted genotypes of HPV 6/11/16/18 in female aged 18-25 years in Australia from 2011 to 2015 (4-8 years post-initiation of HPV NIP). Among 737 vaginal swabs for HPV DNA detection, 184 cases (25.0\%) tested positive for HPV infection. A very low prevalence of qHPV genotypes was observed: $1.7 \%$ in HPV-positive participants.

Dilner et al. [39] estimated the changes in HPV prevalence among $>12,000$ cervical screening female before (2004-2005) and after (2012-2013) the introduction of qHPV vaccination in Denmark, Norway, and Sweden. Despite the small vaccination coverage in cervical screening aged female, the decrease in HPV positive reactions was significant in female aged $18-26$ years, from $54.4 \%$ to $48.1 \%$ $(\mathrm{p}<0.001)$. Moreover, the decrease was significant for $\mathrm{qHPV}$ vaccine types of HPV 6/11/16/18, from $22.3 \%$ to $16.6 \%$ (p $<0.001)$. A significant decrease in $\mathrm{qHPV}$ types was noted in all countries, with a difference of $-6.1 \%$ (from $20.3 \%$ to $14.2 \%$ in Denmark, $\mathrm{p}=0.0005$ ), $-4.1 \%$ (from $26.7 \%$ to $22.6 \%$ in Norway, $\mathrm{p}=0.03$ ), and $-5.6 \%$ (from $19.0 \%$ to $13.4 \%$ in Sweden, $\mathrm{p}=0.0008)$. Furthermore, the nHPV type of HPV 31 also decreased from $8.1 \%$ to $6.4 \%$ (in female aged $18-26$ years) and from $6.4 \%$ to $2.1 \%$ (in female aged $27-50$ years), possibly due to cross-protection from $\mathrm{GHPV}$ vaccination [40].

According to a nationwide study [8] on the annual prevalence of AGW from 2007 to 2015 in Korea, the prevalence in females increased until 2012 and decreased thereafter, with an APC of $+3.6 \%$, highlighting the effect of HPV vaccination (particularly in females) $(p<0.05)$. In contrast, the prevalence in males increased continuously with an APC of $+11.6 \%$, probably due to the decreased HPV vaccination rate compared to females $(\mathrm{p}<0.001)$. As a result, the male-to-female prevalence rate ratios of AGW increased continuously from 1.1-2.0 in all ages over the last nine years, suggested that the prevalence has increased more in males than females $(p<0.001)$

(2) Effectiveness of HPV vaccination in males: Although HPV infections cause diseases and cancers in males, few studies have explored the effectiveness and safety of HPV vaccination in males. According to Harder et al. [41], male vaccination is moderately effective in anogenital HPV infections (efficacy of 46.9\%), grade 2 and 3 anal intraepithelial lesions (61.9\% and 46.8\%, respectively), and oral infections (88\%). Giuliano et al. [42] reported that non-violated qHPV vaccination decreased the incidence of HPV 6/11/16/18-related HPV-associated diseases among 4,065 males in 18 countries. The efficacy of a three-dose regimen qHPV vaccine against HPV 6/11/16/18-related external urogenital lesions was shown to be $90.4 \%$ against condyloma acuminata. The efficacy against persistent infections with HPV6/11/16/18 was $85.6 \%$.

Previous studies suggested that if current vaccination coverage is applied to the male model, it will decrease the HPV-related disease burden to both sexes equally by preventing an additional 22,122 and 25,226 cervical carcinomas and other HPV-associated carcinomas in females over the next 100 years, respectively [4]. HPV vaccination in males prevents $H P V$ infection and the development of associated anogenital lesions. Although the worldwide trend in sex disparity in HPV vaccinations has narrowed, and the vaccine efficacy has been demonstrated in both sexes, HPV vaccine coverage remains lower among males than females in Korea. Scaling up HPV vaccination in males and females in Korea should be encouraged, considering the sex-difference trends in HPV infections.

\section{National Strategy on HPV-Related Disease Prevention in Korea}

In female, cervical cancer screening can prevent cervical cancer as part of the national strategy for HPV infections. The adaptation of screening programs has led to a decreased incidence of cervical cancers worldwide. Many epidemiologic studies have supported the effectiveness of cervical cancer screening on the incidence of invasive squamous cell carcinoma in regions, including the United States, Canada, and Europe [43]. In the United States, NCI-designated cancer centers aimed to screen 93\% of age-eligible females for cervical cancer to provide prompt follow-up and appropriate treatment of females who screen positive for high grade cervical precancerous lesions by 2020 


\section{[34].}

In Korea, the Papanicolaou (Pap) smear was introduced in 1988 in health examinations to employees and those insured by the National Health Insurance (NHI) program. The Pap smear has played an essential role in reducing cervical cancer in Korea. The rate of Pap smears has increased from $33.5 \%$ in 1998 to $45.5 \%$ in 2009 in Korea [43]. Since 2016, the National Cancer Screening Program provides free cervical cancer screening biennially to all Korean female over 20 years of age.

HPV vaccines are recommended in both sexes and are believed to be effective regardless of a previous HPV infection history. Although GNV is provided in some regions, including the United States, Australia, and the United Kingdom, the national strategy of HPV vaccination in Korea continues to target females. GNV is expected to result in greater reductions in HPV-associated disease burdens; hence, HPV vaccination in males should be considered. According to the Korean guidelines for sexually transmitted infections published by the Centers for Disease Control and Prevention, $\mathrm{qHPV}$ or nHPV vaccinations are recommended in males. They are available from the age of 9 years and are recommended between 11-12 years. Adolescents who miss or fail to terminate HPV vaccinations between 9-12 years are recommended to vaccinate from 13-26 years. For males aged 27 or older, qHPV or nHPV are recommended if they have the HPV infection risk factors [44].

\section{CONCLUSIONS}

HPV infections are the most common sexually transmitted infections and an important cause of cervical, anogenital, and oropharyngeal cancers. Although the prevalence of cervical cancer has been decreased markedly owing to the HPV vaccination program in females in many countries, new genotypes, such as HPV 52 and 58, are considered as important as HPV 16, and the prevalence of AGW has been increasing in male in Korea. A new HPV vaccination strategy, such as the GNV program and the introduction of the nHPV vaccine, can eliminate most HPV-related diseases.

\section{CONFLICT OF INTEREST}

No potential conflict of interest relevant to this article was reported.

\section{AUTHOR CONTRIBUTIONS}

Y.J.P. participated in data collection and wrote the manuscript. T.H.K. participated in the study design and coordination. H.H.L. participated in the manuscript editing. J.M.K. participated in the study design and helped to draft the manuscript. All authors read and approved the final manuscript.

\section{ORCID}

Yoo-Jin Park, https://orcid.org/0000-0003-2250-1722

Tae-Hee Kim, https://orcid.org/0000-0003-3478-9102

Hae-Hyeog Lee, https://orcid.org/0000-0003-4876-2912

Jun Mo Kim, https://orcid.org/0000-0003-4978-1867

\section{REFERENCES}

1. Schiffman M, Castle PE, Jeronimo J, Rodriguez AC, Wacholder S. Human papillomavirus and cervical cancer. Lancet 2007;370:890-907.

2. Bodily J, Laimins LA. Persistence of human papillomavirus infection: keys to malignant progression. Trends Microbiol 2011;19:33-9.

3. Herrero R, Castellsagué $X$, Pawlita M, Lissowska J, Kee F, Balaram P, et al.; IARC Multicenter Oral Cancer Study Group. Human papillomavirus and oral cancer: the International Agency for Research on Cancer multicenter study. J Natl Cancer Inst 2003;95:1772-83.

4. Takla A, Wiese-Posselt M, Harder T, Meerpohl JJ, Robl-Mathieu $M$, Terhardt $M$, et al. Background paper for the recommendation of HPV vaccination for boys in Germany. Bundesgesundheitsblatt Gesundheitsforschung Gesundheitsschutz 2018;61:1170-86.

5. Joint Committee on Vaccination and Immunisation. Statement on HPV vaccination [Internet]. London: Joint Committee on Vaccination and Immunisation; c2018 [cited 2018 Jul 18]. Available from: https://assets.publishing.service.gov.uk/ government/uploads/system/uploads/attachment_data/file/72 6319/JCVI_Statement_on_HPV_vaccination_2018.pdf.

6. Brisson M, Benard E, Drolet M, Bogaards JA, Baussano I, Vanska $S$, et al. Population-level impact, herd immunity, and elimination after human papillomavirus vaccination: a systematic review and meta-analysis of predictions from transmission-dynamic models. Lancet Public Health 2016;1:e8-17.

7. Korostil IA, Ali H, Guy RJ, Donovan B, Law MG, Regan DG. Near elimination of genital warts in Australia predicted with extension of human papillomavirus vaccination to males. Sex Transm Dis 2013;40:833-5. 
8. Park YJ, Kim JM, Lee BR, Kim TH, Lee EG. Annual prevalence and economic burden of genital warts in Korea: Health Insurance Review and Assessment (HIRA) service data from 2007 to 2015. Epidemiol Infect 2018;146:177-86.

9. Oh EJ, Jang YM, Cha JY, Kong IS. Sexually transmitted infections (STIs) surveillance in the Republic of Korea, 2014-2018. Public Health Wkly Rep 2020;13:911-21.

10. Jung KW, Won YJ, Kong HJ, Lee ES; Community of Population-Based Regional Cancer Registries. Cancer statistics in Korea: incidence, mortality, survival, and prevalence in 2015. Cancer Res Treat 2018;50:303-16.

11. Nah EH, Cho S, Kim S, Cho HI. Human papillomavirus genotype distribution among 18,815 women in 13 Korean cities and relationship with cervical cytology findings. Ann Lab Med 2017;37:426-33.

12. Bosch FX, Burchell AN, Schiffman M, Giuliano AR, de Sanjose $S$, Bruni $L$, et al. Epidemiology and natural history of human papillomavirus infections and type-specific implications in cervical neoplasia. Vaccine 2008;26 Suppl 10:K1-16.

13. Kreimer AR, Clifford GM, Boyle P, Franceschi S. Human papillomavirus types in head and neck squamous cell carcinomas worldwide: a systematic review. Cancer Epidemiol Biomarkers Prev 2005; 14:467-75.

14. De Vuyst H, Clifford GM, Nascimento MC, Madeleine MM, Franceschi S. Prevalence and type distribution of human papillomavirus in carcinoma and intraepithelial neoplasia of the vulva, vagina and anus: a meta-analysis. Int J Cancer 2009;124:1626-36.

15. Lee EH, Um TH, Chi HS, Hong YJ, Cha YJ. Prevalence and distribution of human papillomavirus infection in Korean women as determined by restriction fragment mass polymorphism assay. J Korean Med Sci 2012;27:1091-7.

16. Quek SC, Lim BK, Domingo E, Soon R, Park JS, Vu TN, et al. Human papillomavirus type distribution in invasive cervical cancer and high-grade cervical intraepithelial neoplasia across 5 countries in Asia. Int J Gynecol Cancer 2013;23:148-56.

17. Park SJ, Seo J, Ha SH, Jung GW. Prevalence and determinants of high-risk human papillomavirus infection in male genital warts. Korean J Urol 2014;55:207-12.

18. Shin HR, Franceschi S, Vaccarella S, Roh JW, Ju YH, Oh JK, et al. Prevalence and determinants of genital infection with papillomavirus, in female and male university students in Busan, South Korea. J Infect Dis 2004;190:468-76.

19. Stratton KL, Culkin DJ. A contemporary review of HPV and penile cancer. Oncology (Williston Park) 2016;30:245-9.

20. Rubin MA, Kleter B, Zhou M, Ayala G, Cubilla AL, Quint WG, et al. Detection and typing of human papillomavirus DNA in penile carcinoma: evidence for multiple independent pathways of penile carcinogenesis. Am J Pathol 2001;159:1211-8.

21. Maden C, Sherman KJ, Beckmann AM, Hislop TG, Teh CZ, Ashley RL, et al. History of circumcision, medical conditions, and sexual activity and risk of penile cancer. J Natl Cancer Inst
$1993 ; 85: 19-24$

22. Dunne EF, Nielson CM, Stone KM, Markowitz LE, Giuliano AR. Prevalence of HPV infection among men: a systematic review of the literature. J Infect Dis 2006;194:1044-57.

23. Giuliano AR, Nielson CM, Flores R, Dunne EF, Abrahamsen M, Papenfuss MR, et al. The optimal anatomic sites for sampling heterosexual men for human papillomavirus (HPV) detection: the HPV detection in men study. J Infect Dis 2007;196: 1146-52.

24. Baldwin SB, Wallace DR, Papenfuss MR, Abrahamsen $M$, Vaught LC, Kornegay JR, et al. Human papillomavirus infection in men attending a sexually transmitted disease clinic. J Infect Dis 2003;187:1064-70.

25. Svare EI, Kjaer SK, Worm AM, Osterlind A, Meijer CJ, van den Brule AJ. Risk factors for genital HPV DNA in men resemble those found in women: a study of male attendees at a Danish STD clinic. Sex Transm Infect 2002;78:215-8.

26. Lazcano-Ponce E, Herrero R, Munoz N, Hernandez-Avila M, Salmeron J, Leyva $A$, et al. High prevalence of human papillomavirus infection in Mexican males: comparative study of penile-urethral swabs and urine samples. Sex Transm Dis $2001 ; 28: 277-80$.

27. Giuliano AR, Lazcano-Ponce E, Villa LL, Flores R, Salmeron J, Lee JH, et al. The human papillomavirus infection in men study: human papillomavirus prevalence and type distribution among men residing in Brazil, Mexico, and the United States. Cancer Epidemiol Biomarkers Prev 2008;17:2036-43.

28. Miralles-Guri C, Bruni L, Cubilla AL, Castellsague X, Bosch FX, de Sanjose S. Human papillomavirus prevalence and type distribution in penile carcinoma. J Clin Pathol 2009;62:870-8.

29. Arbyn M, Castellsague $X$, de Sanjose S, Bruni L, Saraiya M, Bray $\mathrm{F}$, et al. Worldwide burden of cervical cancer in 2008. Ann Oncol 2011;22:2675-86.

30. Kweon SS. Updates on cancer epidemiology in Korea, 2018. Chonnam Med J 2018;54:90-100.

31. Remmerbach TW, Brinckmann UG, Hemprich A, Chekol M, Kuhndel K, Liebert UG. PCR detection of human papillomavirus of the mucosa: comparison between MY09/11 and GP5+/6+ primer sets. J Clin Virol 2004;30:302-8.

32. Bae JH, Lee SJ, Kim CJ, Hur SY, Park YG, Lee WC, et al. Human papillomavirus (HPV) type distribution in Korean women: a meta-analysis. J Microbiol Biotechnol 2008;18:788-94.

33. Patel EU, Grabowski MK, Eisenberg AL, Packman ZR, Gravitt $P E$, Tobian AAR. Increases in human papillomavirus vaccination among adolescent and young adult males in the United States, 2011-2016. J Infect Dis 2018;218:109-13.

34. National Cancer Institute. NCl-designated cancer centers endorse goal of eliminating HPV-related cancers [Internet]. Bethesda: National Cancer Institute; c2018 [cited 2018 Jun 14]. Available from: http://med.stanford.edu/content/dam/sm/ cancer/documents/Banner/Cancer_Center_HPV_Cancer_Elim ination_Statement_FINAL.pdf. 
35. Ouh YT, Min KJ, Cho HW, Ki M, Oh JK, Shin SY, et al. Prevalence of human papillomavirus genotypes and precancerous cervical lesions in a screening population in the Republic of Korea, 2014-2016. J Gynecol Oncol 2018;29:e14.

36. Hintze JM, O'Neill JP. Strengthening the case for gender-neutral and the nonavalent HPV vaccine. Eur Arch Otorhinolaryngol 2018;275:857-65.

37. Machalek DA, Garland SM, Brotherton JML, Bateson D, McNamee K, Stewart M, et al. Very low prevalence of vaccine human papillomavirus types among 18- to 35-year old Australian women 9 years following implementation of vaccination. J Infect Dis 2018;217:1590-600.

38. Garland SM, Cornall AM, Brotherton JML, Wark JD, Malloy MJ, Tabrizi SN; VACCINE study group. Final analysis of a study assessing genital human papillomavirus genoprevalence in young Australian women, following eight years of a national vaccination program. Vaccine 2018;36:3221-30.

39. Dillner J, Nygard M, Munk C, Hortlund M, Hansen BT, Lagheden $\mathrm{C}$, et al. Decline of HPV infections in Scandinavian cervical screening populations after introduction of HPV vaccination programs. Vaccine 2018;36:3820-9.

40. Wheeler CM, Kjaer SK, Sigurdsson K, Iversen OE,
Hernandez-Avila $M$, Perez $G$, et al. The impact of quadrivalent human papillomavirus (HPV; types 6, 11, 16, and 18) L1 virus-like particle vaccine on infection and disease due to oncogenic nonvaccine HPV types in sexually active women aged 16-26 years. J Infect Dis 2009; 199:936-44.

41. Harder T, Wichmann O, Klug SJ, van der Sande MAB, Wiese-Posselt M. Efficacy, effectiveness and safety of vaccination against human papillomavirus in males: a systematic review. BMC Med 2018;16:110.

42. Giuliano AR, Palefsky JM, Goldstone S, Moreira ED Jr, Penny $M E$, Aranda $C$, et al. Efficacy of quadrivalent HPV vaccine against HPV infection and disease in males. $N$ Engl J Med 2011;364:401-11.

43. Oh CM, Jung KW, Won YJ, Shin A, Kong HJ, Jun JK, et al. Trends in the incidence of in situ and invasive cervical cancer by age group and histological type in Korea from 1993 to 2009. PLoS One 2013;8:e72012.

44. Korea Centers for Disease Control and Prevention, Korean Association of Urogenital Tract Infection and Inflammation. Korean sexually transmitted infection guidelines 2016. 2nd ed. Cheongju: Korea Centers for Disease Control and Prevention; 2016. 